\title{
CONTINUOUS SELECTION OF REPRESENTING MEASURES ${ }^{1}$
}

\author{
BY H. S. BEAR
}

Communicated by Victor Klee, August 25, 1969

Let $B$ be a linear subspace of $C_{R}(X)$, the continuous real functions on a compact space $X$, and let $\Gamma$ be the Silov boundary of $B$ in $X$. We give here conditions which are sufficient for there to be an integral representation of the form

$$
u(x)=\int_{\Gamma} u(\theta) g_{x}(\theta) d \mu(\theta)
$$

where $x \rightarrow g_{x}$ is a continuous map from some subset $\Delta$ of $X$ into $L_{\infty}(\mu)$. With the additional condition that $\Delta$ is separable, we obtain a kernel representation of the form

$$
u(x)=\int_{\Gamma} u(\theta) Q(x, \theta) d \mu(\theta)
$$

where $Q$ is a continuous function of $x$ and $x \rightarrow Q(x, \cdot)$ is continuous with respect to the $L_{\infty}(\mu)$ norm. If it is also the case that $B \mid \Gamma$ is dense in $L_{1}(\mu)$, then $Q(\cdot, \theta)$ is a limit (uniform convergence on compact subsets of $\Delta$ ) of functions in $B$. These results also give integral representations like (1) and (2) for a complex function algebra, simply by considering the space $B$ of real parts of the algebra. The details of this work will appear in [3].

We use the following notation throughout this paper:

$X$ is a compact Hausdorff space, with topology $\mathfrak{J}$.

$B$ is a linear subspace of $C_{R}(X)$, containing the constant functions, and separating the points of $X$.

$\Gamma$ is the Silov boundary of $B$ in $X$.

$\bar{B}_{\Delta}$, for any set $\Delta \subset X$, is the closure of $B \mid \Delta$ in the topology of uniform convergence on compact subsets of $\Delta$.

$B^{+}(\Delta, z)=\{u \mid \Delta: u \in B, u>0, u(z)=1\}$.

$\left(\bar{B}_{\Delta}\right.$ is an abstract version of "all harmonic functions on $\Delta$," and $B^{+}(\Delta, z)$ is the set of normalized-at-z positive $B$-functions.)

AMS Subject Classifications. Primary 4625, 4655; Secondary 5200.

Key Words and Phrases. Linear subspace of $C_{R}(X)$, integral representation kernel representation, Gleason parts, part metric, function algebra.

1 This research was supported in part by grant NSF GP 7681. 
A representing measure $\nu$ for $x \in X$ is a positive Baire probability measure on $\Gamma$ such that $u(x)=\int_{\Gamma} u d \nu$ for all $u \in B$.

$P_{x}$ is the set of all representing measures for $x$.

$B^{\perp}$ is the set of real signed measures on $\Gamma$ which are orthogonal to $B$.

$B^{\prime}$ is the space of sup-norm continuous linear functionals on $B$, with the $w^{*}$-topology $=$ the $w\left(B^{\prime}, B\right)$ topology $=$ the topology of pointwise convergence on $B$.

$T_{B}=\left\{F \in B^{\prime}: F(1)=\|F\|=1\right\} . T_{B}$ is a compact convex set in $B^{\prime}$.

We consider $X$ embedded homeomorphically in $T_{B}$. Then $\Gamma$ is the closure of the extreme points of $T_{B}$, and $B$ is isometric to the restriction to $T_{B}$ or $X$ or $\Gamma$ of all $w^{*}$-continuous linear functionals on $B^{\prime}$.

We use $x, y, z$ etc. for points of $T_{B}$, and write $u(x)$ for $x(u)$, where $x \in T_{B}$ and $u \in B$.

Write $x \sim y$ for $x, y \in T_{B}$ iff there is a number $a$ such that

$$
a^{-1}<u(x) / u(y)<a
$$

for all strictly positive $u \in B$. The relation $\sim$ is an equivalence relation, and the equivalence classes are called the Gleason parts of $X$ or $T_{B}$. The parts of $X$ are the intersections of $X$ with the parts of $T_{B}$. The Gleason parts of a complex function algebra $A$ are the same as the parts defined by the space $B=\operatorname{Re} A$.

On each part $\Delta$ of $X$ or $T_{B}$ define a metric as follows:

$d(x, y)=\sup \{|\log u(x)-\log u(y)|: u \in B, u>0\}$. Write $d(x, y)=\infty$ if $x x y$. Let $J_{d}$ be the $d$-metric topology on $\Delta$.

The parts of $T_{B}$ are convex subsets which can be characterized as follows: $x \sim y$ iff the segment $[x, y]$ extends some distance beyond $x$ and $y$ in $T_{B}$; i.e., if

$$
\begin{aligned}
& (1+r) x-r y \in T_{B}, \\
& (1+r) y-r x \in T_{B},
\end{aligned}
$$

for some $r>0$. Then we also have

$$
d(x, y)=\inf \{\log (1+1 / r): r \text { satisfies }(4)\} .
$$

We use this definition of part and part metric $d$ in any convex set containing no whole line.

Let $C=C_{R}(\Gamma)$, so that $T_{C}$ can be identified with the positive probability measures on $\Gamma$. The part $\Pi_{\mu}$ of $T_{C}$ containing $\mu$ is the set of all measures $g \mu$, where $g$ is a positive function of $L_{\infty}(\mu)$ which is bounded away from zero.

We use $D$ for the part metric in parts of $T_{C}$. Convergence in $D$ is equivalent to convergence of the corresponding $g$ 's in $L_{\infty}(\mu)$. We write $D(g, h)$ instead of $D(g \mu, h \mu)$ for points $g \mu, h \mu$ of $T_{c}$. 
The part metric on any part of a function space is complete. That is $d$ is complete on any part $\Delta$ of $X$ or $T_{B}$, and $D$ is complete on parts $\Pi_{\mu}$ of $T_{C}$.

Let $\Delta$ be a part of $X$ with part metric $d$. Then $\Im=J_{d}$ iff $B^{+}(\Delta, z)$ is equicontinuous for some (every) $z \in \Delta$.

The following theorem indicates the extent to which our sufficient conditions are necessary.

THEOREM 1. If $\Delta$ is a subset of $X$, and there is a continuous map $x \rightarrow g_{x} \mu$ on $\Delta$ to a part $\Pi_{\mu}$ of $T_{c}$ (so that $x \rightarrow g_{x}$ is a continuous map into $\left.L_{\infty}(\mu)\right)$ and

$$
u(x)=\int_{\Gamma} u g_{x} d \mu
$$

for all $u \in B, x \in \Delta$, then $\Delta$ is contained in a part of $X$, and $J=J_{d}$ on $\Delta$.

For any convex set $K$, let $K^{i}$ be the set of all $x \in K$ such that for every $y \in K$, the segment $[y, x]$ extends beyond $x$ in $K$. If $K^{i} \neq 0$, then $K^{i}$ is a part, which we call the inner part of $K$. (Incidentally, $K$ is a convex body in some linear topological space iff $K^{i} \neq 0$.) Finite dimensional convex sets have nonempty inner parts.

Theorem 2. Let $\Delta$ be a part of $X$, with $J=J_{d}$ on $\Delta$. Let $P_{x}^{i} \neq 0$ for some $x \in \Delta$. Then there is a continuous map $x \rightarrow g_{x} \mu$ on $\Delta$ into some part $\Pi_{\mu}$ of $T_{C}$ such that $g_{x} \mu \in P_{x}$ for all $x \in \Delta$.

Note. The condition $\Im=J_{d}$ can be replaced by the assumption that $B^{+}(\Delta, z)$ is equicontinuous. The condition $P_{x}^{i} \neq 0$ is implied by the assumption that $B^{\perp}$ is finite dimensional. The measure $g_{x} \mu$ is a $D$ continuous function of $x$, which implies that $g_{x}$ is an $L_{\infty}$-continuous function of $x$.

INDICATION OF PROOF. If $B^{\perp}$ is finite dimensional, then each $P_{x}$ is, and hence $P_{x}^{i} \neq 0$. If $P_{x}^{i} \neq 0$ for some $x$, then $P_{y}^{i} \neq 0$ for all $y \sim x$, and the sets $P_{x}^{i}, x \in \Delta$, are all contained in one part $\Pi_{\mu}$ of $T_{c}$. This result is due to Har'kova [6], who makes ingeneous use of Bishop's theorem [5]. If $\hat{\Delta}$ is the part of $T_{B}$ containing $\Delta$, then $P_{x}^{i} \subset \Pi_{\mu}$ for all $x \in \hat{\Delta}$. The projection of $\Pi_{\mu}$ into $T_{B}$ (restriction of the measures from $C=C_{R}(\Gamma)$ to $B$ ) is a $D-d$ continuous affine map on $\Pi_{\mu}$ onto $\hat{\Delta}$. This restriction map is therefore open, since $\Pi_{\mu}, D$ and $\hat{\Delta}, d$ are one-part complete convex sets [1]. The inverse map, $x \rightarrow P_{x} \cap \Pi_{\mu}=P_{x}^{i}$, is a lower semicontinuous map of $\hat{\Delta}$ into $D$-closed convex subsets of $\Pi_{\mu}$. By an adaptation of Michael's continuous selection theorem to part-metric setting [2, Theorem 12] there is a continuous selection $x \rightarrow g_{x} \mu \in P_{x}^{i}$. Continuity with respect to $d$ on $\Delta$ and $D$ in $\Pi_{\mu}$ is the same as con- 
tinuity with respect to $J$ on $\Delta$, and the $L_{\infty}$ norm applied to the functions $g_{x}$.

TheOREM 3. Assume the rypotheses of Theorem 2, and in addition that $\Delta$ has a countable dense set. Then there is a measure $\mu$ on $\Gamma$ and a jointly measurable function $Q(x, \theta)$ on $\Delta \times \Gamma$ such that $Q(x, \cdot) d \mu(\cdot)$ represents $x, x \rightarrow Q(x, \cdot)$ is $L_{\infty}$-continuous, and $Q(\cdot, \theta)$ is continuous on $\Delta$ for each $\theta \in \Gamma$. If $B \mid \Gamma$ is dense in $L_{1}(\mu)$, then $Q(\cdot, \theta) \in \bar{B}_{\Delta}$ for each $\theta \in \Gamma$.

Proof. The proof of [4] can be modified to get from the measureable functions $g_{x}(\theta)$ to a kernel $Q(x, \theta)$ in the separable case. The fact that $Q(\cdot, \theta) \in \bar{B}_{\Delta}$ is also proved in [4], with an assumption that implies $B \mid \Gamma$ is dense in $L_{1}(\mu)$.

\section{REFERENCES}

1. Heinz Bauer, An open mapping theorem for convex sets with only one part (to appear).

2. Heinz Bauer and H. S. Bear, The part metric in convex sets, Pacific J. Math. 30 (1969), 15-33.

3. H. S. Bear, Lectures on Gleason parts, Springer Lecture Notes (to appear).

4. H. S. Bear and Bertram Walsh, Integral kernel for one-part function spaces, Pacific J. Math. 23 (1967), 209-215. MR 36 \#6643.

5. Errett Bishop, Representing measures for points in a uniform algebra, Bull. Amer. Math. Soc. 70 (1964), 121-122. MR 28 \#1510.

6. N. V. Har'kova, Generalized Poisson formula, Vestnik Muskov Univ. Ser. I Mat. Meh. 22 (1967), no. 4, 25-30. (Russian) MR 35 \#4333.

University of Hawait, Honolulu, Hawair 96822 